[8] M. Abramovici, M. A. Breuer, and A. D. Friedman, Digital Systems Testing and Testable Design. Piscataway, NJ: IEEE Press, 1995.

[9] I. Pomeranz and S. M. Reddy, "Forward-looking fault simulation for improved static compaction," IEEE Trans. Comput.-Aided Design Integr. Circuits Syst., vol. 20, no. 10, pp. 1262-1265, Oct. 2001.

[10] X. Lin, J. Rajski, I. Pomeranz, and S. M. Reddy, "On static test compaction and test pattern ordering for scan designs," in Proc. Int. Test Conf., Oct. 2001, pp. 1088-1097.

[11] A. H. El-Maleh and Y. E. Osais, "Test vector decomposition-based static compaction algorithms for combinational circuits," ACM Trans. Des. Autom. Electron. Syst., vol. 8, no. 4, pp. 430-459, Oct. 2003.

[12] S. C. Ma, P. Franco, and E. J. McCluskey, "An experimental chip to evaluate test techniques experiment results," in Proc. Int Test Conf., 1995, pp. 663-672.

[13] S. M. Reddy, I. Pomeranz, and S. Kajihara, "Compact test sets for high defect coverage," IEEE Trans. Comput.-Aided Design Integr. Circuits Syst., vol. 16, no. 8, pp. 923-930, Aug. 1997.

[14] J. T.-Y. Chang, C.-W. Tseng, C.-M. J. Li, M. Purtell, and E. J. McCluskey, "Analysis of pattern-dependent and timing-dependent failures in an experimental test chip," in Proc. Int. Test Conf., 1998, pp. 184-193.

[15] J. Dworak, M. R. Grimaila, S. Lee, L.-C. Wang, and M. R. Mercer, "Enhanced DO-RE-ME based defect level prediction using defect site aggregation-MPG-D," in Proc. Int. Test Conf., 2000, pp. 930-939.

[16] I. Pomeranz and S. M. Reddy, "Definitions of the numbers of detections of target faults and their effectiveness in guiding test generation for high defect coverage," in Proc. Conf. Des. Autom. Test Eur., 2001, pp. 504-508.

[17] B. Benware, C. Schuermyer, N. Tamarapalli, K.-H. Tsai, S. Ranganathan, R. Madge, J. Rajski, and P. Krishnamurthy, "Impact of multipledetect test patterns on product quality," in Proc. Int. Test Conf., 2003, pp. 1031-1040.

[18] S. Venkataraman, S. Sivaraj, E. Amyeen, S. Lee, A. Ojha, and R. Guo, "An experimental study of $n$-detect scan ATPG patterns on a processor," in Proc. VLSI Test Symp., 2004, pp. 23-28.

[19] H. Tang, G. Chen, S. M. Reddy, C. Wang, J. Rajski, and I. Pomeranz, "Defect aware test patterns," in Proc. Des. Autom. Test Eur. Conf., 2005, pp. $450-455$.

[20] I. Pomeranz and S. M. Reddy, "Forming $N$-detection test sets without test generation," ACM Trans. Des. Autom. Electron. Syst., vol. 12, no. 2, pp. 1-18, Apr. 2007. Article 18.

[21] S. Sengupta, S. Kundu, S. Chakravarty, P. Parvathala, R. Galivanche, G. Kosonocky, M. Rodgers, and T. M. Mak, "Defect-based tests: A key enabler for successful migration to structural test," Intel Technol. J., vol. Q.1, 1999.

[22] V. Krishnaswamy, A. B. Ma, and P. Vishakantaiah, "A study of bridging defect probabilities on a Pentium (TM) 4 CPU," in Proc. Int. Test Conf., 2001, pp. 688-695.

\section{Quadratic Backward Propagation of Variance for Nonlinear Statistical Circuit Modeling}

\author{
Ivica Stevanović, Member, IEEE, and \\ Colin C. McAndrew, Fellow, IEEE
}

\begin{abstract}
Accurate statistical modeling and simulation are keys to ensure that integrated circuits (ICs) meet the specifications over the stochastic variations that are inherent in IC manufacturing technologies. Backward propagation of variance (BPV) is a general technique for statistical modeling of semiconductor devices. However, the BPV approach assumes that statistical fluctuations are not large so that variations in device electrical performances can be modeled as linear functions of process parameters. With technology scaling, device performance variability over manufacturing variations becomes nonlinear. In this paper, we extend the BPV technique to take into account these nonlinearities. We present the theory behind the technique and apply it to specific examples. We also investigate the effectiveness of several possible solution algorithms.
\end{abstract}

Index Terms-Backward propagation of variance (BPV), nonlinear devices, semiconductor device modeling, statistical modeling.

\section{INTRODUCTION}

Integrated circuit (IC) design in the presence of manufacturing process variations requires statistical simulation [1], [2] and, therefore, statistical device models [3]. The models should work with all types of statistical analyses: process corner simulation, distributional (Monte Carlo or MC) simulation, and mismatch simulation.

A number of different approaches for statistical modeling have been proposed. The simplest method is to introduce variations directly into Simulation Program with Integrated Circuit Emphasis (SPICE) model parameters; however, this leads to inaccurate results as it ignores correlations between SPICE model parameters.

Extreme case data, either from split manufacturing lots or from physical simulations of manufacturing extremes, can be used to generate models [4]. However, this does not give distributional models. Standard numerical techniques like principle components analysis [5]-[7] and response surface modeling [8]-[10] can be used to generate statistical models. However, these require significant effort to extract model parameter sets from many statistical device samples and can neither be updated efficiently if a process changes, as it does during development, nor be used for prediction for new generation technologies.

Given a process and a geometry level model, statistical modeling can be done by directly measuring the statistics of the process level model parameters $\boldsymbol{p}$ and then basing the statistical SPICE models on these variations [11], [12]. Although this provides both corner and distributional models, it is not always possible to directly measure all required process parameters. More importantly, this does not take into account the fact that the same variation in similar parameters for different models will give different variations in the modeled electrical performances $e$ [3]. The goal of modeling is to accurately represent

Manuscript received April 18, 2008; revised November 7, 2008 and March 11, 2009. Current version published August 19, 2009. This paper was recommended by Associate Editor D. Sylvester.

I. Stevanović was with the Freescale Semiconductor, 1211 Geneva, Switzerland. He is now with the ABB Corporate Research, 5405 BadenDättwil, Switzerland, and also with the Electromagnetics and Acoustics Laboratory, Ecole Polytechnique Fédérale de Lausanne, 1015 Lausanne, Switzerland (e-mail: ivica.stevanovic@ch.abb.com; ivica.stevanovic@epfl.ch).

C. C. McAndrew is with the Freescale Semiconductor, Tempe, AZ 85284 USA (e-mail: colin.mcandrew @ freescale.com).

Digital Object Identifier 10.1109/TCAD.2009.2023194 
the device electrical performance variation, not the model parameter variation.

A general technique for statistical modeling of semiconductor devices, called backward propagation of variance (BPV), was introduced in [3], [13], and [14]. This technique is based on the models defined as functions of the fundamental (independent) process parameters $\boldsymbol{p}$ (used for statistical simulation for IC design) and on the statistical device data collected in manufacturing. The method is termed BPV because it takes measurements in variances of important electrical quantities and then calculates the variances in the process parameters necessary to fit the measured data. This gives models that are guaranteed to match the manufacturing extremes and distributions of $\boldsymbol{e}$ (including mismatch). In addition, the models can easily be updated when the process changes or new generation technologies are introduced. However, BPV assumes that statistical variations are not large so that linearization of $\boldsymbol{e}(\boldsymbol{p})$ is accurate. For general measures of circuit performance, this is not always valid [15], and even for common measures of device performances, this assumption is becoming less valid because variability increases as device dimensions continue to decrease.

In this paper, we extend the BPV technique to account for the nonlinearities in $\boldsymbol{e}(\boldsymbol{p})$. We denote this technique "quadratic BPV" (QBPV) as it is based on the quadratic (forward) propagation of variance approach introduced in [16]. We present the theory behind the technique and its application to specific examples. We also investigate the effectiveness of several possible solution algorithms.

\section{Propagation of VARiance}

Consider an electrical performance $e_{i}=e_{i}(\boldsymbol{p})$, where $\boldsymbol{p}=\left(p_{1}\right.$, $\left.p_{2}, \ldots, p_{N}\right)$ is the vector of $N$ process parameters. The seconddegree Taylor series expansion of $e_{i}$ in the neighborhood of $\overline{\boldsymbol{p}}=$ $\left(\bar{p}_{1}, \bar{p}_{2}, \ldots, \bar{p}_{N}\right)$ gives

$$
e_{i}(\boldsymbol{p}) \approx e_{i}(\overline{\boldsymbol{p}})+\sum_{j=1}^{N}\left(p_{j}-\bar{p}_{j}\right)\left(\sum_{k=1}^{N} s_{i, j}+s_{i, j k}\left(p_{k}-\bar{p}_{k}\right)\right)
$$

where

$$
s_{i, j}=\left.\frac{\partial e_{i}(\boldsymbol{p})}{\partial p_{j}}\right|_{\boldsymbol{p}=\overline{\boldsymbol{p}}} \quad s_{i, j k}=\left.\frac{1}{2} \frac{\partial^{2} e_{i}(\boldsymbol{p})}{\partial p_{j} \partial p_{k}}\right|_{\boldsymbol{p}=\overline{\boldsymbol{p}}} .
$$

If $p_{j}$ is statistically independent and normally distributed with $p_{j} \sim \mathcal{N}\left(\bar{p}_{j}, \sigma_{j}^{2}\right)$, then it follows that [16], [17]

$$
\begin{aligned}
& \mu_{e_{i}}=\mathrm{E}\left\{e_{i}\right\} \approx e_{i}(\overline{\boldsymbol{p}})+\sum_{j=1}^{N} s_{i, j j} \sigma_{j}^{2} \\
& \sigma_{e_{i}}^{2}=\operatorname{var}\left\{e_{i}\right\} \approx \sum_{j=1}^{N} s_{i, j}^{2} \sigma_{j}^{2}+2 \sum_{j, k=1}^{N} s_{i, j k}^{2} \sigma_{j}^{2} \sigma_{k}^{2} .
\end{aligned}
$$

For $s_{i, j k}=0$, this gives the standard linear form

$$
\mu_{e_{i}}=\mathrm{E}\left\{e_{i}\right\} \approx e_{i}(\overline{\boldsymbol{p}}) \quad \sigma_{e_{i}}^{2}=\operatorname{var}\left\{e_{i}\right\} \approx \sum_{j=1}^{N} s_{i, j}^{2} \sigma_{j}^{2} .
$$

The standard linear form (4) is sufficiently accurate if $e_{i}$ is only weakly nonlinear in the neighborhood of $\overline{\boldsymbol{p}}$. For electrical performances that are not well approximated by the linear functions of the process parameters, a better approximation uses the quadratic forms (2) and (3). In contrast to the linear approximation, $\mathrm{E}\left\{e_{i}\right\}$ is no longer equal to $e_{i}(\overline{\boldsymbol{p}})$; therefore, $e_{i}$ is no longer normally distributed.
The measure of the degree of asymmetry (deviation from normality) of the probability distribution function of $e_{i}$ is the skewness [18]

$$
\gamma_{e_{i}}=\mu_{e_{i}^{3}}^{3} / \mu_{e_{i}^{2}}^{3 / 2}
$$

where $\mu_{e^{n}}=\mathrm{E}\left\{\left(e_{i}-\mathrm{E}\left\{e_{i}\right\}\right)^{n}\right\}$ denotes the $n$ th-order central moments of the distribution of $e_{i}$. After some algebraic manipulation

$$
\gamma_{e_{i}} \approx \frac{1}{\sigma_{e_{i}}^{3}} \sum_{j, k=1}^{N} s_{i, j k} \sigma_{j}^{2} \sigma_{k}^{2}\left(6 s_{i, j} s_{i, k}+8 \sum_{l=1}^{N} s_{i, k l} s_{i, l j} \sigma_{l}^{2}\right)
$$

from which it is apparent that, for a linear response $\left(s_{i, j k}=0\right)$, the skewness of $e_{i}$ is zero, as expected.

\section{QBPV}

The statistical variation in a particular process parameter $p_{j}$ can be modeled as

$$
p_{j}=\bar{p}_{j}+\delta p_{j}
$$

where $\bar{p}_{j}$ is the nominal (mean) value of the parameter and $\delta p_{j}$ is its variation. We assume that all process parameter variations are normally distributed and statistically independent. (A parameter that is distributed lognormally, i.e., the nonideal base-emitter saturation current for bipolar transistors $I_{\text {ben }}$, can be modeled by introducing a normally distributed statistical parameter and multiplying $I_{\text {ben }}$ by the exponential of that statistical parameter. Parameters that are partially correlated, for example, channel length variation for NMOS and PMOS devices, can be modeled by using appropriate combinations of independent parameters [19]. We have yet to encounter any statistical device modeling problem that, with some thought, cannot be formulated based on independent normally distributed process parameters.)

Let $\boldsymbol{e}=\left(e_{1}, e_{2}, \ldots, e_{M}\right)$ be a vector of the device electrical performances that can be measured in IC manufacturing lines. Statistical variations in $\boldsymbol{p}$ directly affect the variations in $e$. Rather than calculating the variances of $\boldsymbol{e}$ from the variances of $\boldsymbol{p}$ using (4), for statistical modeling purposes, the procedure can be reversed to characterize the variances of $\boldsymbol{p}$ from the measured variances of $\boldsymbol{e}$. If $\sigma_{e_{i}}$ is known, then $s_{i, j}=\partial e_{i} / \partial p_{j}$ can be calculated about the nominal value $\overline{\boldsymbol{p}}$ from the underlying SPICE models. The variance equations of (4) then give a set of linear equations that can be solved for the unknowns $\sigma_{j}$. This procedure is termed BPV [3]. (The nominal values of $\boldsymbol{p}$, which give the nominal values of $e$, are determined using nonlinear least squares optimization. The sensitivities are computed from the $\pm 3 \sigma$ variations in each $p_{j}$; therefore, the solution for $\sigma_{j}$ is done iteratively until the sensitivities and the standard deviations are self-consistent.)

Note that not all process parameters $p_{j}$ need to be characterized statistically using BPV [3]. For example, for MOSFETs, it can be best to directly measure the distribution of the oxide thickness from the capacitance data. Therefore, statistical characterization can be a mix of forward and backward propagations of variance. If the variances of $n$ of the process parameters $p_{j}, j \in \mathcal{F}=\left\{j_{1}, j_{2}, \ldots, j_{n}\right\}$, are known, then (4) becomes

$$
\sigma_{e_{i}}^{2}-\sum_{j \in \mathcal{F}} s_{i, j}^{2} \sigma_{j}^{2}=\sum_{j \in \mathcal{B}} s_{i, j}^{2} \sigma_{j}^{2}, \quad i=1, \ldots, M
$$

where $\mathcal{B}=\{j=1,2, \ldots, N: j \notin \mathcal{F}\}$ is the set of indexes of the BPV process parameters. These equations can be solved as long as $M \geq N-n$.

For situations where approximation (4) is not sufficiently accurate, the statistical modeling problem then becomes: How can the nominal values and variances of $p_{j}$ be calculated so that the nominal values, 
variances, and skewness of $e_{i}$ are modeled accurately? We propose a new approach, i.e., the QBPV, for this task.

If $n$ process parameters are Forward Propagation of Variance (FPV) parameters, then, from (2)

$$
\mu_{e_{i}}-e_{i}(\overline{\boldsymbol{p}})-\sum_{j \in \mathcal{F}} s_{i, j j} \sigma_{j}^{2}=\sum_{j \in \mathcal{B}} s_{i, j j} \sigma_{j}^{2} .
$$

The mean value of $\delta e_{i}=e_{i}-e_{i}(\overline{\boldsymbol{p}})$ is not zero since a nonlinear function of expected values is not equal to the expected value of the nonlinear function. Therefore, the processes of finding the mean values and the variances of the process parameters cannot be anymore decoupled, as is the case for the BPV procedure. The first $M$ of the QBPV system of equations is, therefore, (9).

Accounting for the FPV parameters, from (3), one obtains

$$
\begin{aligned}
\sigma_{e_{i}}^{2}-\sum_{j \in \mathcal{F}}\left(s_{i, j}^{2} \sigma_{j}^{2}+2 s_{i, j j}^{2} \sigma_{j}^{4}\right) \\
\quad=\sum_{j \in \mathcal{B}}\left(s_{i, j}^{2}+4 \sum_{k \in \mathcal{F}} s_{i, j k}^{2} \sigma_{k}^{2}\right) \sigma_{j}^{2}+2 \sum_{j, k \in \mathcal{B}} s_{i, j k}^{2} \sigma_{j}^{2} \sigma_{k}^{2} .
\end{aligned}
$$

These provide additional $M$ equations that must be solved as part of the QBPV procedure.

The final set of $M$ equations for the QBPV process targets the fitting of the skewness of the electrical performances. From (6)

$$
\begin{aligned}
\gamma_{e_{i}} \sigma_{e_{i}}^{3} & -\sum_{j \in \mathcal{F}}\left(6 s_{i, j}^{2} s_{i, j j} \sigma_{j}^{4}+8 s_{i, j j}^{3} \sigma_{j}^{6}\right) \\
= & 12 \sum_{j \in \mathcal{B}} \sum_{k \in \mathcal{F}}\left(s_{i, j} s_{i, k}+2 s_{i, j k} s_{i, k k} \sigma_{k}^{2}\right) s_{i, j k} \sigma_{j}^{2} \sigma_{k}^{2} \\
& +6 \sum_{j, k \in \mathcal{B}}\left(s_{i, j} s_{i, k}+4 \sum_{m \in \mathcal{F}} s_{i, j m} s_{i, k m} \sigma_{m}^{2}\right) s_{i, j k} \sigma_{j}^{2} \sigma_{k}^{2} \\
& +8 \sum_{j, k, l \in \mathcal{B}} s_{i, j k} s_{i, k l} s_{i, l j} \sigma_{j}^{2} \sigma_{k}^{2} \sigma_{l}^{2} .
\end{aligned}
$$

The QBPV equations (9)-(11) give $3 M$ equations to solve for the $2(N-n)$ unknowns, i.e., the means and the variances of the BPV process parameters $p_{j}, j \in \mathcal{B}$. The defined modeling targets are the means, the variances, and the skewnesses of each of the $M$ electrical performances.

\section{NUMERICAL RESUlTS}

\section{A. Three Methods to Numerically Solve the QBPV Equations}

We have a system of $3 M$ equations (for the mean, variance, and skewness of each device performance) with at most $2 N$ unknowns (the unknown means and variances of the process parameters). The first $M$ equations (9) have $\bar{p}_{j}$ coupled with $\sigma_{j}$ as the unknowns. The remaining $2 M$ equations (10) and (11) are the nonlinear functions of $\sigma_{j}$. These equations all involve sensitivity coefficients that depend on the underlying SPICE models and, more importantly, on the unknown values $\bar{p}_{j}$ (as for a nonlinear function, the sensitivity $s_{i, j}=\partial e_{i} / \partial p_{j}$ depends on the point $\boldsymbol{p}$ at which it is evaluated). Therefore, in contrast to the (linear) BPV process, where the means $\bar{p}_{j}$ can be determined as one step and the sensitivity and variance computations can be done as independent and subsequent steps, for QBPV, the means and the sensitivities need to be calculated self-consistently with, and coupled to, the solution for $\sigma_{j}$. We have investigated three methods to solve the QBPV equations (see Fig. 1).

Method 1: Obtain initial estimates for the means and variances of the process parameters, for example, from linear BPV. From these
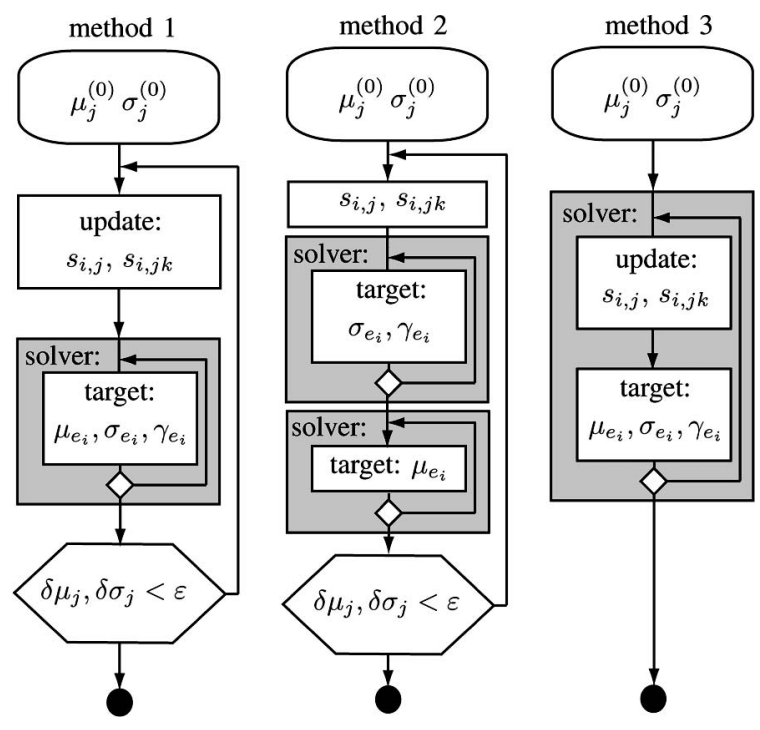

Fig. 1. Three methods to numerically solve the QBPV equations.

values, compute the (first and second order) sensitivity coefficients $s_{i, j}$ and $s_{i, j k}$. Based on these sensitivities, use a nonlinear solver to compute the means and the variances of $p_{j}$ that best satisfy (9)-(11). Iterate the sensitivity calculations and subsequent computations of the means and variances of $p_{j}$ until they are, within some convergence criterion, self-consistent.

Method 2: Obtain initial estimates for the means and variances of the process parameters, for example, from linear BPV. From these values, compute the (first and second order) sensitivity coefficients $s_{i, j}$ and $s_{i, j k}$. Based on these sensitivities, use a nonlinear solver to compute the variances of $p_{j}$ that best satisfy (10) and (11). Use these variances in (9) to update values for the process parameter means, and, then, recalculate the sensitivities. Iterate the sequential variance and mean calculations until they converge.

Method 3: Obtain initial estimates for the means and variances of the process parameters, for example, from linear BPV. Use a nonlinear (iterative) solver to self-consistently solve the system of equations (9)-(11). Here, "self-consistent" means that, as the means and the standard deviations of the process parameters change at each step of the iterative solution process, the calculations of the sensitivity coefficients, which are based on the perturbations of $\pm 3 \sigma$ of each $p_{j}$, are updated at each iteration. Although this takes more computation time per iteration than methods 1 and 2, for the sensitivity calculations, it obviates the need for the outer iteration loop of methods 1 and 2 and could improve the overall convergence.

For both methods 1 and 2, damping is used for the outer loop iterations on both the means and the variances. Among the software packages for solving systems of nonlinear equations and nonlinear least square problems, TENSOLVE [20], which is based on the tensor method, is proven to be robust and efficient [20], [21] and is used here.

\section{B. Evaluation of Convergence Properties}

To evaluate the methods, we first investigate a known analytical nonlinear problem with $N=3$ process parameters $p_{j}$ and $M=3$ device performances $e_{i}$ related by the following system of equations:

$$
e_{i}=\sum_{j=1}^{N} a_{i j}^{2} p_{j}^{2}
$$

This nonlinear example is quadratic; therefore, the QBPV procedure should give an exact solution. It can be shown that the $e_{i}$ for this 
TABLE I

Convergence Properties of THE THree QBPV SOlution Methods For Different Initial Values of the Process Parameter Sigma. (A) $\sigma_{j}=0.1$. (B) $\sigma_{j}=1.1$. (C) $\sigma_{j}=2$. (D) $\sigma_{j}=10$

\begin{tabular}{|c|c|c|c|c|c|c|}
\cline { 2 - 7 } \multicolumn{1}{c|}{} & \multicolumn{2}{c|}{ Method 1 } & \multicolumn{2}{c|}{ Method 2 } & \multicolumn{2}{c|}{ Method 3 } \\
\cline { 2 - 7 } \multicolumn{1}{c|}{} & \# iterations & CPU (s) & \# iterations & CPU (s) & \# iterations & CPU (s) \\
\hline A & 45 & 587 & 13 & 28.6 & 6 & 61.2 \\
\hline B & 47 & 302 & 13 & 28.6 & 6 & 60.9 \\
\hline C & fail & - & 13 & 28.8 & 6 & 60.7 \\
\hline D & fail & - & 13 & 28.7 & 6 & 60.9 \\
\hline
\end{tabular}

example has mean, variance, and skewness given by

$$
\begin{aligned}
\mu_{e_{i}} & =\sum_{j} a_{i j}^{2}\left(\mu_{j}^{2}+\sigma_{j}^{2}\right) \\
\sigma_{e_{i}}^{2} & =2 \sum_{j} a_{i j}^{4} \sigma_{j}^{2}\left(2 \mu_{j}^{2}+\sigma_{j}^{2}\right) \\
\gamma_{e_{i}} & =\frac{8}{\sigma_{e_{i}}^{3}} \sum_{j} a_{i j}^{6} \sigma_{j}^{4}\left(3 \mu_{j}^{2}+\sigma_{j}^{2}\right) .
\end{aligned}
$$

We choose $\mu_{p_{j}}=\sigma_{p_{j}}=0.5 j$ and $a_{i j}=(i+j) / 10$, where $i=1$, $\ldots, M$ and $j=1, \ldots, N$. This gives the following target mean, sigma, and skewness values: $\mu_{e_{i}}=\{0.92,1.49,2.2\}, \quad \sigma_{e_{i}}=$ $\{0.9093,1.4336,2.0788\}$, and $\gamma_{e_{i}}=\{2.0170,1.9778,1.9491\}$, $i=1,2,3$. Using these target values, we applied the QBPV procedure to calculate the means and the variances of the process parameters, using each of the three methods described previously. The default TENSOLVE convergence tolerances, which depend on the machine precision [20], were used, the maximum number of TENSOLVE iterations was 40 , and the maximum number of "outer loop" iterations for methods 1 and 2 was 60 . For these two double-iteration procedures, the convergence of the outer loop was defined as when the change in the process parameter means and variances between two consecutive iterations was less than $0.01 \%$. Table I summarizes the convergence and the CPU time results (for methods 1 and 2, the number of iterations is of the outer loop; for method 3, it is the number of TENSOLVE iterations). Note that, for each iteration for method 3, the sensitivity coefficients are updated about six times.

Methods 2 and 3 are the most robust and converge equally well for the different initial values that were tried. Method 3 needs on average only six TENSOLVE iterations to converge, but each iteration takes approximately $10 \mathrm{~s}$ because of the effort needed to compute the sensitivities. In contrast, method 2 needs 13 outer loop iterations to converge, but each iteration takes significantly less time, i.e., about $2 \mathrm{~s}$, because there is only one sensitivity calculation per iteration. Method 1 is the least robust in terms of convergence (for initial value cases $\mathrm{C}$ and $\mathrm{D}$, it did not converge after a maximum of 60 iterations).

\section{Evaluation of Solution Accuracy}

In this section, we investigate the performance of methods 2 and 3 in solving the analytical quadratic example when the target values are given exactly as computed from the analytical formulas. We also investigate the performance of QBPV when only the mean and the variance are targeted, as well as the mean, the variance, and the skewness, and compare the results with those obtained from the linear BPV procedure (which only targets the mean and the variance).

When targeting the values of an analytic nonlinear problem, both methods 2 (Table II) and 3 (Table III) perform equally well. This is valid when we target the mean and the variances only and when we target the skewness values as well (i.e., when we have an overdetermined system). Moreover, the QBPV methods converge, as expected, to the
TABLE II

RESUlts OBTAINED USING BPV AND QBPV (METHOD 2)

\begin{tabular}{|c|c|c|c|c|c|c|}
\cline { 2 - 7 } \multicolumn{1}{c|}{} & \multicolumn{2}{c|}{ BPV } & \multicolumn{2}{c|}{ QBPV $(\mu, \sigma)$} & \multicolumn{2}{c|}{ QBPV $(\mu, \sigma, \gamma)$} \\
\cline { 2 - 7 } \multicolumn{1}{c|}{} & $\mu$ & $\sigma$ & $\mu$ & $\sigma$ & $\mu$ & $\sigma$ \\
\hline$p_{1}$ & 0.7071 & 0.4328 & 0.5001 & 0.5000 & 0.5001 & 0.5000 \\
\hline$p_{2}$ & 1.4142 & 0.8660 & 1.0001 & 0.9999 & 1.0000 & 0.9999 \\
\hline$p_{3}$ & 2.1213 & 1.2990 & 1.5002 & 1.4999 & 1.5002 & 1.4999 \\
\hline residual (\#iter.) & \multicolumn{2}{|c|}{$10^{-23}(2)$} & $10^{-5}(22)$ & $10^{-6}(13)$ \\
\hline
\end{tabular}

TABLE III

RESUlts OBTAINED UsING BPV AND QBPV (METHOD 3)

\begin{tabular}{|c|c|c|c|c|c|c|}
\cline { 2 - 7 } \multicolumn{1}{c|}{} & \multicolumn{2}{c|}{ BPV } & \multicolumn{2}{c|}{ QBPV $(\mu, \sigma)$} & \multicolumn{2}{c|}{ QBPV $(\mu, \sigma, \gamma)$} \\
\cline { 2 - 7 } \multicolumn{1}{c|}{} & $\mu$ & $\sigma$ & $\mu$ & $\sigma$ & $\mu$ & $\sigma$ \\
\hline$p_{1}$ & 0.7071 & 0.4328 & 0.5000 & 0.5000 & 0.5000 & 0.5000 \\
\hline$p_{2}$ & 1.4142 & 0.8660 & 1.0000 & 1.0000 & 1.0000 & 1.0000 \\
\hline$p_{3}$ & 2.1213 & 1.2990 & 1.5000 & 1.5000 & 1.5000 & 1.5000 \\
\hline residual (\#iter.) & \multicolumn{2}{|c|}{$10^{-23}(2)$} & \multicolumn{2}{c|}{$10^{-16}(5)$} & \multicolumn{2}{c}{$10^{-15}(6)$} \\
\hline
\end{tabular}

\begin{tabular}{|c|c|c|c|c|c|c|c|}
\hline & \multicolumn{2}{|c|}{ BPV Model } & & \multicolumn{2}{|c|}{ MC (BPV Model) } & \multicolumn{2}{|c|}{ Fab. Data } \\
\hline & $\mu$ & $\sigma$ & & $\mu$ & $\sigma$ & $\mu$ & $\sigma$ \\
\hline$\rho_{\mathrm{bs}}$ & 0.6512 & $20.21 \%$ & $\beta$ & 82.6 & $43.80 \%$ & 80.0 & $30.65 \%$ \\
\hline$J_{\text {bei }}$ & 0.7777 & $18.27 \%$ & $V_{\text {bes }}$ & $0.8515 \mathrm{~V}$ & $9.20 \mathrm{mV}$ & $0.85 \mathrm{~V}$ & $10.03 \mathrm{mV}$ \\
\hline$\Delta L_{\mathrm{e}}$ & -0.0019 & 0.0781 & $V_{\mathrm{af}}$ & $49.3 \mathrm{~V}$ & $28.88 \%$ & $46.4 \mathrm{~V}$ & $39.17 \%$ \\
\hline
\end{tabular}

TABLE IV

BJT RESULTS OBTAINED USING BPV

\begin{tabular}{|c|c|c|c|c|c|c|c|}
\hline & \multicolumn{2}{|c|}{ QBPV Model } & & \multicolumn{2}{|c|}{ MC (QBPV Model) } & \multicolumn{2}{|c|}{ Fab. Data } \\
\hline & $\mu$ & $\sigma$ & & $\mu$ & $\sigma$ & $\mu$ & $\sigma$ \\
\hline$\rho_{\mathrm{bs}}$ & 0.6922 & $19.77 \%$ & $\beta$ & 79.3 & $28.37 \%$ & 80.0 & $30.65 \%$ \\
\hline$J_{\text {bei }}$ & 0.8461 & $18.02 \%$ & $V_{\text {bes }}$ & $0.8498 \mathrm{~V}$ & $9.26 \mathrm{mV}$ & $0.85 \mathrm{~V}$ & $10.03 \mathrm{mV}$ \\
\hline$\Delta L_{\mathrm{e}}$ & 0.0010 & 0.0788 & $V_{\mathrm{af}}$ & $45.6 \mathrm{~V}$ & $37.80 \%$ & $46.4 \mathrm{~V}$ & $39.17 \%$ \\
\hline
\end{tabular}

TABLE V

BJT RESULTS OBTAINED USING QBPV

known values of the process parameters, whereas the BPV procedure, because it assumes linear $e_{i}(\boldsymbol{p})$ mappings, has some inaccuracy.

\section{BJT Example}

In this section, we analyze the QBPV procedure on an n-p-n bipolar junction transistor (BJT). The models used included mappings from geometry and process parameters to model parameters [12], [13]. The $\boldsymbol{p}$ 's used for the statistical modeling were the following: the (normalized) pinched base-sheet resistance $\rho_{\mathrm{bs}}$, the (normalized) ideal component of base current density $J_{\text {bei }}$, and the geometric deviation in the emitter length $\Delta L_{e}$. The $e$ 's targeted for the statistical modeling were the following: the current gain $\beta=I_{c} / I_{b}$, the base-emitter voltage $V_{\text {bes }}$ (measured at a fixed emitter current with the base and the collector tied together), and the forward Early voltage $V_{\text {af }}$ (calculated from the collector current measured at two collector voltages and fixed base voltage).

The measured means and standard deviations of the device electrical performances from the fab data are in the last columns of Table IV. The results of the BPV procedure (computed values for the means and standard deviations of $\boldsymbol{p}$ ) and the statistics from a 10000 -sample MC simulation based on the BPV model results are in the remaining columns. Table V shows a similar model and the MC simulation results from the QBPV procedure solved using method 2. (The BPV procedure took eight iterations to self-consistently solve for the standard deviations of $\boldsymbol{p}$; the QBPV procedure took 11 iterations. The asextracted model was from silicon that was different from the typical one for the process; hence, the nominal values (means) of the process 

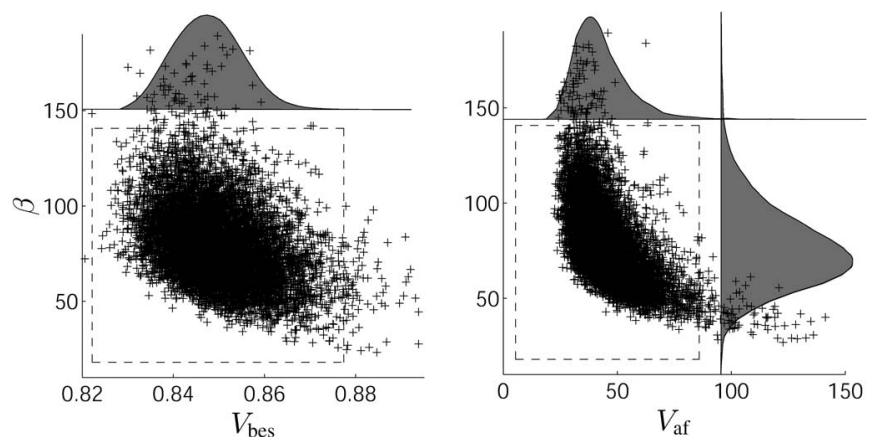

Fig. 2. Scatter plots of the BJT device performances from the MC simulations. The dashed lines are the $\pm 3 \sigma$ limits; the shaded bell curves are the sample distributions.

parameters had to be skewed as part of the statistical model generation to center the model on the target fab data.)

Comparison of the measured values with the MC simulations shows that the QBPV results are in better agreement with the measurements than the BPV results. This is expected, because the $\boldsymbol{e}(\boldsymbol{p})$ mappings are fairly nonlinear for BJTs, and the variations are reasonably large. That this problem is nonlinear was confirmed from the skewnesses computed from the MC simulation $\left(\gamma_{\beta}=1.2, \gamma_{V_{\mathrm{bes}}}=0.7\right.$, and $\left.\gamma_{V_{\mathrm{af}}}=4.2\right)$ and is visually apparent in the scatter plots of the MC results (see Fig. 2).

\section{CONCLUSION}

BPV is a common technique to characterize process parameter variability. This method is effective if the random process parameter deviations are normally distributed and statistically independent and if the dependence of the device electrical performances on the process parameters is nearly linear. With technology scaling and increased variability of process parameters, the assumption of linearity is no longer always reasonable. In this paper, we have introduced QBPV that takes into account the nonlinear dependences of the device electrical performances on the process parameters. We have investigated several different techniques for solving the QBPV equations and compared them in terms of accuracy and computational efficiency. QBPV has been applied to a set of nonlinear problems and provides a significant improvement in the accuracy compared with the (linear) BPV procedure. The method is important in practice as it helps in the reduction of parametric yield loss and overdesign in high-variability and nonlinear IC processes.

\section{REFERENCES}

[1] W. Maly and A. J. Strojwas, "Statistical simulation of the IC manufacturing process," IEEE Trans. Comput.-Aided Design Integr. Circuits Syst., vol. CAD-1, no. 3, pp. 120-131, Jul. 1982.

[2] S. R. Nassif, "Modeling and forecasting of manufacturing variations," in Proc. Int. Workshop Stat. Metrology, Honolulu, HI, Jun. 11, 2000, pp. 2-10.
[3] C. C. McAndrew, "Efficient statistical modeling for circuit simulation," in Design of System on a Chip: Devices \& Components, R. Reis and J. Jess, Eds. Norwell, MA: Kluwer, 2004, pp. 97-122.

[4] E. J. Prendergast and P. Lloyd, "A highly automated integrated modeling system: Mecca," in Proc. IEEE Custom Integr. Circuits Conf., Portland, OR, May 20-22, 1985, pp. 516-519.

[5] C. K. Chow, "Projection of circuit performance distributions by multivariate statistics," IEEE Trans. Semicond. Manuf., vol. 2, no. 2, pp. 60-65, May 1989.

[6] M. Bolt, M. Rocchi, and J. Engel, "Realistic statistical worst-case simulations of VLSI circuits," IEEE Trans. Semicond. Manuf., vol. 4, no. 3, pp. 193-198, Aug. 1991.

[7] J. A. Power, B. Donnellan, A. Mathewson, and W. A. Lane, "Relating statistical MOSFET model parameter variabilities to IC manufacturing process fluctuations enabling realistic worst case design," IEEE Trans. Semicond. Manuf., vol. 7, no. 3, pp. 306-318, Aug. 1994.

[8] J. A. Power, S. C. Kelly, E. C. Griffith, D. Doyle, and M. O'Neill, "Statistical modeling for a $0.6 \mu \mathrm{m}$ BiCMOS technology," in Proc. IEEE Bipolar/ BiCMOS Circuits Technol. Meeting., Minneapolis, MN, Sep. 28-30, 1997, pp. 24-27.

[9] K. Singhal and V. Visvanathan, "Statistical device models from worst case files and electrical test data," IEEE Trans. Semicond. Manuf., vol. 12, no. 4, pp. 470-484, Nov. 1999.

[10] A. A. Mutlu and M. Rahman, "Statistical methods for the estimation of process variation effects on circuit operation," IEEE Trans. Electron. Packag. Manuf., vol. 28, no. 4, pp. 364-375, Oct. 2005.

[11] P. Cox, P. Yang, S. S. Mahant-Shetti, and P. Chatterjee, "Statistical modeling for efficient parametric yield estimation of MOS VLSI circuits," IEEE Trans. Comput.-Aided Design Integr. Circuits Syst., vol. CAD-32, no. 2, pp. 471-478, Feb. 1985.

[12] W. F. Davis and R. T. Ida, "Statistical IC simulation based on independent wafer extracted process parameters and experimental designs," in Proc. IEEE Bipolar Circuits Technol. Meeting, Minneapolis, MN, Sep. 18-19, 1989, pp. 262-265.

[13] C. C. McAndrew, J. Bates, R. T. Ida, and P. Drennan, "Efficient statistical BJT modeling, why $\beta$ is more than $I_{\mathrm{c}} / I_{\mathrm{b}}$," in Proc. IEEE Bipolar/ BiCMOS Circuits Technol. Meeting, Minneapolis, MN, Sep. 28-30, 1997, pp. $28-31$.

[14] C. C. McAndrew, "Statistical modeling for circuit simulation," in Proc. IEEE Int. Symp. Qual. Electron. Des., San Jose, CA, Mar. 24-26, 2003, pp. 357-362.

[15] J. Ogrodzki, L. Opalski, and M. Styblinski, "Acceptability regions for a class of linear networks," in Proc. IEEE Int. Symp. Circuits Syst., Houston, TX, Apr. 28-30, 1980, pp. 187-190.

[16] J. Lei, P. Lima-Filho, and M. A. Styblinski, "Quadratic propagation of variance (QPOV) in statistical circuit design," in Proc. Int. Workshop Stat. Metrology, Honolulu, HI, Jun. 7, 1998, pp. 88-91.

[17] A. Graupner, Q. Schwarz, and R. Schüffny, "Statistical analysis of analog structures through variance calculation," IEEE Trans. Circuits Syst. I, Fundam. Theory Appl., vol. 49, no. 8, pp. 1071-1078, Aug. 2002.

[18] D. Zwillinger, Ed., CRC Standard Mathematical Tables and Formulae, 31st ed. Boca Raton, FL: CRC Press, 2003.

[19] C. C. McAndrew and P. G. Drennan, "Device correlation: Modeling using uncorrelated parameters, characterization using ratios and differences," in Proc. NSTI Nanotechnol. WCM, Boston, MA, May 9-11, 2006, pp. 698-702.

[20] A. Bouaricha and R. B. Schnabel, "Algorithm 768: TENSOLVE: A software package for solving systems of nonlinear equations and nonlinear least-squares problems using tensor methods," ACM Trans. Math. Softw., vol. 23, no. 2, pp. 174-195, Jun. 1997.

[21] D. Dent, M. Paprzycki, and A. Kucaba-Pietal, "Recent advances in solvers for nonlinear equations," Comput. Assist. Mech. Eng. Sci., vol. 7, pp. $493-$ $505,2000$. 